\title{
A VOLUME COMPARISON THEOREM AND NUMBER OF ENDS FOR MANIFOLDS WITH ASYMPTOTICALLY NONNEGATIVE RICCI CURVATURE
}

\author{
Mahaman BAZANFARÉ
}

\begin{abstract}
In this paper we establish a volume comparison theorem for cocentric metric balls at arbitrary point for manifolds with asymptotically nonnegative Ricci curvature, which will allow us to prove the finiteness of the number of ends
\end{abstract}

\section{Introduction}

In (CG1) and (CG2) J.Cheeger and D.Gromoll studied noncompact manifolds with nonnegative (sectional and Ricci) curvature and showed they have finite topology.

In 1985 U.Abresch in [Ab1] and [Ab2] introduced a new concept: manifolds with asymptotically nonnegative sectional curvature. A noncompact manifold of dimension $\mathrm{m}$ is said to be with asymptotically nonnegative sectional curvature (Ricci curvature) if there exists a point $\mathrm{p}$, called base point and a positive, nonincreasing function $\lambda$ so that:

$$
\int_{0}^{+\infty} t \lambda(t) d t=b_{0}<+\infty
$$

and for all $x \in M$ and $u$ in $T_{x} M$

$$
K(u) \geq-\lambda(d(p, x))\|u\|^{2} \quad\left(\operatorname{Ricci}(u) \geq-(m-1) \lambda(d(p, x))\|u\|^{2}\right) .
$$

One of the remaining questions is if asymptotically nonnegative curvature implies finiteness of number of ends. U.Abresch and D.Gromoll in 1991 Mathematics Subject Classification: 53C20.

Servicio de Publicaciones. Universidad Complutense. Madrid, 2000 
[AG] showed that if $M$ has asymptotically nonnegative curvature and has diameter growth of order $o\left(r^{\frac{1}{n}}\right)$ then it is homotopically equivalent to an interior of compact manifold with boundary, provided the sectional curvature is bounded from below. M.I.Cai in [Ca] established a bound on number of ends of manifolds with nonnegative Ricci curvature outside a compact subset. S.H.Zhu in [Zh] proved a volume comparison theorem for balls with center the base point in manifolds with asymptotically nonnegative Ricci curvature.

The purpose of this paper is to establish a comparison theorem at every point, not necessary the base point and deduce a finite bound on the number of ends. In the second theorem we show that manifolds with asymptotically nonnegative Ricci curvature have infinite volume.

Let take some definitions .

Two curves $\gamma_{1}$ and $\gamma_{2}$ on a riemannian manifold $M$ with origin x are said to be cofinal if and only if for all ball $\mathrm{B}(\mathrm{x}, \mathrm{r})$, there exists $t>0$ so that $\gamma_{1}\left(t_{1}\right)$ and $\gamma_{2}\left(t_{2}\right)$ lie in the same connected component of $M / \overline{B(x, r)}$ for all $t_{1}, t_{2} \geq t$ where $\overline{B(x, r)}$ denotes the closed ball of center $x$ and radius $r$.

In this way, an equivalence relation is defined and every class is called an end of $M$.

Let $r>0$ and $B(p, r)$ the ball of center $p$ and radius $r$; we note $\mathcal{C}(p, r)$ the union of all connected unbounded components of $M / \overline{B(p, r)}$. Let $\zeta$ be a real, $\frac{1}{2}<\zeta<1, \Omega$ an open set in $M$ and $\Sigma$ a connected subset of $\Omega$; for $x, y \in \Sigma \cap S(p, r)$ let:

$$
d_{r}(x, y)=i n f_{\gamma} L(\gamma)
$$

where infinum is taken over all smooth curves $\gamma$ in $\Sigma$ from $x$ to $y$. We set

$$
\operatorname{diam}(\Sigma \cap S(p, r), \Omega)=\sup _{(x, y) \in \Sigma} d_{r}(x, y) .
$$

and

$$
\operatorname{Diam}(p, r)=\operatorname{Supdiam}(\Sigma \cap S(p, r), \mathcal{C}(p, \zeta r)),
$$

where suprenum is taken over all unbounded connected components $\Sigma$ of $\partial \mathcal{C}(p, r)$. The function $\operatorname{Diam}(p, r)$ measures the diameter of ends.

The author would like to express deep gratitude to Professor M. Françoise Roy for his continuous encouragement. 


\section{Main Results}

Theorem 1. Let $M$ be a complete open Riemannian manifold with dimension $m$ and asymptotically nonnegative Ricci curvature. Then, for all point $x$ in $M$ and for all $0<r \leq R$

$$
\begin{aligned}
& \frac{\operatorname{Vol}(B(x, R))}{\operatorname{Vol}(B(x, r))} \leq e^{(m-1) b_{0}}\left(\frac{R}{r}\right)^{m} \text { if } 0 \leq R \leq l \\
& \frac{\operatorname{Vol}(B(x, R))}{\operatorname{Vol}(B(x, r))} \leq e^{(m-1) b_{0}}\left(\frac{R+l}{r}\right)^{m} \text { if } R \geq l
\end{aligned}
$$

where $B(x, r)$ denotes the ball of radius $r$ with center $x$ in $M$.

Corollary. Let $M$ be a manifold with asymptotically nonnegative Ricci curvature with base point $p$. Then,

$$
\operatorname{Diam}(p, r) \leq 4 \xi e^{(m-1) b_{0}}\left(1+\frac{3}{\xi}\right) r .
$$

where $\xi=\frac{1}{2}(1-\zeta)$ and consequently $M$ has finite number of ends. This corollary shows that the diameter of ends of an asymptotically nonnegative curved manifold grown at most lineary as on nonnegative curved manifolds.

Theorem 2. Let $M$ be a manifold with asymptotically nonnegative Ricci curvature with base point $p$. Then, there exist two positive constants $C$ and $\rho$ such that,for all $R>0$

$$
\operatorname{Vol}(B(p, R)) \geq C(\ln R)^{\rho} .
$$

For all $t \geq 0$, let $\alpha(t)=\lambda(|t-l|)$ where $l=d(p, x)$. If $z \in M$ and $u \in T_{z} M$ then, by triangle inequality, we have:

$$
|d(x, z)-d(x, p)| \leq d(p, z)
$$

and since $\lambda$ is nonincreasing,

$$
\operatorname{Ric}_{z}(u) \geq-(m-1) \lambda(d(p, z)) \geq-(m-1) \alpha(d(x, z))
$$




\section{Proofs}

To prove our results we need three lemmas:

Lemma1. Let $y(t)$ be the unique solution of equation:

$$
(*)\left\{\begin{array}{l}
y^{\prime \prime}(t)-\alpha(t) y(t)=0 \\
y(0)=0, y^{\prime}(0)=1
\end{array}\right.
$$

then for all $t \geq 0, t \leq y(t) \leq c e^{b_{0}}$.t where

$$
c= \begin{cases}1 & \text { if } t \leq l \\ \left(1+2 l \lambda_{1}(0)\right) e^{b_{0}} & \text { if } t \geq l\end{cases}
$$

where $\lambda_{1}(0)=\int_{0}^{\infty} \lambda(x) d x$

Proof. Since $y^{\prime \prime}(t)=\alpha(t) y(t) \geq 0, y^{\prime}$ is increasing in a neighbourhood of zero and one easily shows like in [Zh] that $y(t) \geq t$ for all $t \geq 0$ and that $y$ is increasing.

By initial conditions, we have:

$$
\begin{gathered}
y^{\prime}(t)=1+\int_{0}^{t} \alpha(s) y(s) d s \Longrightarrow \\
y(t)=t+\int_{0}^{t}\left(\int_{0}^{s} \alpha(x) y(x) d x\right) d s=t+\int_{0}^{t}\left(\int_{x}^{t} \alpha(x) y(x) d s\right) d x
\end{gathered}
$$

by Fubini.

$$
\begin{gathered}
y(t)=t+\int_{0}^{t}(t-x) \alpha(x) y(x) d x=t\left(1+\int_{0}^{t} \alpha(x) y(x) d x\right)-\int_{0}^{t} x \alpha(x) y(x) d x \\
\Longrightarrow y(t) \geq t\left(1+\int_{0}^{t} \alpha(x) y(x) d x\right)-t y(t) \int_{0}^{t} \alpha(x) d x \\
\Longrightarrow 1+t \int_{0}^{t} \alpha(x) d x \geq \frac{t y^{\prime}(t)}{y(t)}(* *)
\end{gathered}
$$

If $l=0$ inequality holds by theorem 2.1 in [Zh]

Suppose $l>0$.

If $t \leq l$ then $(* *)$ implies

$$
\frac{1}{t}+\int_{0}^{t} \alpha(x) d x \geq \frac{y^{\prime}(t)}{y(t)} .
$$


After integrating this expression we have

$$
\begin{gathered}
\Longrightarrow \ln \frac{t}{\epsilon}+\int_{\epsilon}^{t} \int_{0}^{x} \lambda(l-u) d u d x \geq \ln \frac{y(t)}{y(\epsilon)} \\
\Longrightarrow \ln y(t) \leq \ln \frac{t y(\epsilon)}{\epsilon}+\int_{\epsilon}^{t} \int_{0}^{x} \lambda(l-u) d u d x
\end{gathered}
$$

By the initial conditions, we have

$$
\begin{gathered}
\ln y(t) \leq \ln t+\int_{\epsilon}^{t} \int_{0}^{x} \lambda(l-u) d u d x \leq \ln y(t) \leq \ln t+\int_{0}^{t} \int_{0}^{x} \lambda(l-u) d u d x \\
\Longrightarrow \ln y(t) \leq \ln t+\int_{0}^{t} \int_{u}^{t} d x \lambda(l-u) d u \\
\Longrightarrow \ln y(t) \leq \ln t+\int_{0}^{t}(t-u) \lambda(l-u) d u \leq \ln t+\int_{0}^{l} u \lambda(u) d u \\
\leq \ln t+b_{0}
\end{gathered}
$$

that is

$$
y(t) \leq e^{b_{0}} \cdot t
$$

If $t \geq l$ we have:

$$
\begin{gathered}
y^{\prime}(t)=y^{\prime}(l)+\int_{l}^{t} \alpha(x) y(x) d x \\
\Longrightarrow y(t)-y(l)=(t-l) y^{\prime}(l)+\int_{l}^{t} \int_{l}^{x} y(u) \lambda(u-l) d u d x \\
=(t-l) y^{\prime}(l)+\int_{l}^{t}(t-u) \lambda(u-l) y(u) d u \\
\leq(t-l)\left(y^{\prime}(l)+\int_{l}^{t} \lambda(u-l) y(u) d u\right)=(t-l) y^{\prime}(t) .
\end{gathered}
$$

then

$$
\frac{\alpha(t) y(t)}{y^{\prime}(t)} \leq(t-l) \alpha(t)+\frac{\alpha(t) y(l)}{y^{\prime}(t)}
$$

hence

$$
\ln \frac{y^{\prime}(t)}{y^{\prime}(l)} \leq \int_{l}^{t}(x-l) \alpha(x)+\int_{l}^{t} \frac{\alpha(x) y(l) d x}{y^{\prime}(l)+\int_{l}^{x} \alpha(u) y(u) d u}
$$




$$
\begin{aligned}
& \ln \frac{y^{\prime}(t)}{y^{\prime}(l)} \leq b_{0}+\int_{l}^{t} \frac{\alpha(x) y(l) d x}{y^{\prime}(l)+y(l) \int_{l}^{x} \alpha(u) d u} \\
& \ln \left(\frac{y^{\prime}(t)}{y^{\prime}(l)}\right) \leq b_{0}+\ln \left(\frac{y^{\prime}(l)+y(l) \lambda_{1}(0)}{y^{\prime}(l)}\right)
\end{aligned}
$$

From (**) we have

$$
\begin{aligned}
y^{\prime}(l) & \leq y(l)\left(\frac{1}{l}+\lambda_{1}(0)\right) \leq e^{b_{0}}\left(1+l \lambda_{1}(0)\right) \\
& \Longrightarrow y^{\prime}(t) \leq e^{2 b_{0}} \cdot\left(1+2 l \lambda_{1}(0)\right)
\end{aligned}
$$

hence

$$
y(t) \leq e^{2 b_{0}}\left(1+2 l \lambda_{1}(0)\right) t
$$

and the lemma follows. The lemma1 is far from giving a sharp comparison for volume of balls with center $x \neq p$ and a sufficient large radius. The basic fact on this is related to the choice of the function $\alpha$. So, we state the following lemma to prove our comparison theorem:

Lemma 2. Let $\bar{M}$ be a noncompact simply connected manifold with dimension $m$. Suppose there exists a point $\bar{p}$ so that

$$
K(\bar{x})=-\lambda(d(\bar{p}, \bar{x}))
$$

for all $\bar{x} \in \bar{M}$. Then, for all $R>0$ and $\bar{x}$, we have 0

$$
\operatorname{Vol}\left(B(\bar{p}, R) \leq \omega_{m} R^{m} e^{(m-1) b_{0}}\right.
$$

$$
\operatorname{Vol}(B(\bar{x}, r)) \geq \omega_{m} r^{m}
$$

and consequently

$$
\text { (3) } \quad \frac{\operatorname{Vol}(B(\bar{x}, R)}{\operatorname{Vol}(B(\bar{x}, r)} \leq \begin{cases}e^{(n-1) b_{0}}\left(\frac{R}{r}\right)^{m} & \text { if } r \leq R \leq l \\ e^{(n-1) b_{0}}\left(\frac{R+l}{r}\right)^{m} & \text { if } R \geq l\end{cases}
$$

where $\omega_{m}$ is the volume of the unit ball in euclidian space. 


\section{Proof}

For all $R>0$ we have

$$
\operatorname{Vol}\left(B(\bar{p}, R)=\int_{S^{n-1}} \int_{0}^{R} y^{m-1}(t) d t d \theta\right.
$$

By lemma 1 we have $y(t) \leq e^{b_{0}} t(l=0)$ and the conclusion follows. Since $K(\bar{x}) \leq 0$ for all $\bar{x}$, the inequality (2) follows from Rauch inequality and the fact that $\bar{M}$ is simply connected. .

If $R \geq l$ we have

$$
B(\bar{x}, R) \subset B(\bar{p}, R+l)
$$

and inequality (3) follows from (1) and (2).

The follwing lemma was proved in [Zh] (lemma2.2)

Lemma 3. Let $f$ and $g$ be two positive functions defined over $[0,+\infty[$. If $f / g$ is nonincreasing, then for $R \geq r>0$ we have:

$$
\frac{\int_{0}^{R} f(t) d t}{\int_{0}^{r} f(t) d t} \leq \frac{\int_{0}^{R} g(t) d t}{\int_{0}^{r} g(t) d t} .
$$

\section{Proof of theorem 1}

Let $J$ denote the Jacobian of exponential application in polar coordinates and the nonnegative function $z$ so that $z^{m-1}=J$; J. Cheeger showed in [Ch] that $z$ satisfies the inequation:

$$
\left\{\begin{array}{l}
z^{\prime \prime}(t)-\alpha(t) z(t) \leq 0 \\
z(0)=0, z^{\prime}(0)=1
\end{array}\right.
$$

from which it follows that $\frac{z}{y}$ is nonincreasing where $y$ is the solution of (*) and by the lemma2 we conclude that

$$
\begin{aligned}
\frac{V o l(B(x, R))}{\operatorname{Vol}(B(x, r))}= & \frac{\int_{0}^{\min \{c u t(\theta), R\}} J(t) d t}{\int_{0}^{\min \{\operatorname{cut}(\theta), r\}} J(t) d t} \leq \frac{\int_{0}^{\min \{\operatorname{cut}(\theta), R\}} y^{m-1}(t) d t}{\int_{0}^{\min \{\operatorname{cut}(\theta), r\}} y^{m-1}(t) d t} \\
& \leq \frac{\int_{0}^{R} y^{m-1}(t) d t}{\int_{0}^{r} y^{m-1}(t) d t}=\frac{\operatorname{Vol}(B(\bar{x}, R)}{\operatorname{Vol}(B(\bar{x}, r))}
\end{aligned}
$$




$$
\leq e^{(m-1) b_{0}}\left(\frac{R+l}{r}\right)^{m}
$$

where $\operatorname{cut}(\theta)$ means the cut point of $\theta$

\section{Proof of corollary}

If $r>0$ and $\left\{q_{j}\right\}$ is the maximal set of points on $S(p, r)$ so that the balls $B\left(q_{j}, \xi r\right)$ are disjoint and are contain in $M / B(p, \zeta r)$. We have :

$$
B\left(q_{j}, \xi . r\right) \subset B(p,(1+\xi) \cdot r) \subset B\left(q_{j},(2+\xi) \cdot r\right)
$$

then

$$
\frac{\operatorname{Vol}(B(p,(1+\xi) \cdot r))}{\operatorname{Vol}\left(B\left(q_{j}, \xi \cdot r\right)\right)} \leq \frac{\operatorname{Vol}\left(B\left(q_{j},(2+\xi) \cdot r\right)\right)}{\operatorname{Vol}\left(B\left(q_{j}, \xi \cdot r\right)\right)} \leq e^{(m-1) b_{0}}\left(\frac{3+\xi}{\xi}\right)^{m},
$$

therefore, the number of balls $B\left(q_{j}, \xi r\right)$ is no more than $e^{(m-1) b_{0}}\left(1+\frac{3}{\xi}\right)^{m}$. The balls $B\left(q_{j}, 2 \xi . r\right)$ cover $S(p, r)$ and if $\gamma$ is a geodesic in $S(p, r)$ joining two points $q_{i}$ and $q_{j}$ so that $B\left(q_{j}, 2 \xi . r\right) \cap B\left(q_{i}, 2 \xi . r\right)$ is not empty then $L(\gamma) \leq 4 \xi r$, hence

$$
\operatorname{Diam}(p, r) \leq 4 \xi e^{(m-1) b_{0}}\left(1+\frac{3}{\xi}\right)^{m} r .
$$

Let $\left\{\gamma_{i}\right\}$ be the set of all geodesics from the base point $p$; if $x_{1}=\gamma_{1}((1+\xi) r), x_{2}=\left(\gamma_{2}((1+\xi) r)\right.$ are in two different connected components of

$M \overline{B(p, r)}$ and if $\theta$ is a short geodesic joining $x_{1}$ to $x_{2}$, then $\theta$ meets $\overline{B(p, r)}$ and

$$
\begin{gathered}
d\left(\gamma_{1}(1+\xi) r, \gamma_{2}(1+\xi) r\right) \geq d\left(\gamma_{1}(1+\xi) r, B(p, r)\right)+d\left(B(p, r), \gamma_{2}(1+\xi) r\right) \\
\geq 2 \xi . r,
\end{gathered}
$$

hence the ball $\overline{B\left(q_{j}, \xi . r\right)}$ contains at most one $\gamma_{k}(1+\xi r)$ which means that the number of ends is less or equal to $e^{(m-1) b_{0}}\left(\frac{3+\xi}{\xi}\right)^{m}$.

Theorem2 states that, as for open complete manifolds with nonnegative Ricci curvature, the open complete manifolds with asymptotically nonnegative Ricci curvature have no finite volume. 


\section{Proof of theorem 2}

Since $M$ is noncompact and complete, for all $t>0$ there exists a point $x$ in $M$ so that $d(p, x) \geq t$. Let $\gamma$ be geodesic arc joining $p$ to $x$, the function $s \longmapsto d(p, \gamma(s))$ is continuous and takes the value t. Let $b>1$ , $R_{i}=\sum_{j=0}^{i} 2 r_{j}$ and $r_{i}=2^{b^{i}}$. Take a point $x_{i}$ on $\gamma$ so that $d\left(p, x_{i}\right)=$ $r_{i}+R_{i-1}$ and $x_{0}=p$; by construction the balls $B\left(x_{i}, r_{i}\right)$ are disjoint and

$$
\bigcup_{j=0}^{i} B\left(x_{j}, R_{j}\right) \subset B\left(x_{i}, r_{i}+R_{i-1}\right) .
$$

Let

$$
\theta_{i}=\sum_{j=0}^{i} \operatorname{volB}\left(x_{j}, r_{j}\right)
$$

then

$$
\begin{gathered}
\theta_{i} \leq \operatorname{volB}\left(x_{i}, r_{i}+R_{i-1}\right) \\
\frac{\theta_{i}}{\theta_{i}-\theta_{i-1}} \leq \frac{\operatorname{volB}\left(x_{i}, r_{i}+R_{i-1}\right)}{\operatorname{volB}\left(x_{i}, r_{i}\right)} \leq e^{(m-1) b_{0}}\left(1+\frac{R_{i-1}}{r_{i}}\right)^{m}
\end{gathered}
$$

since $d\left(p, x_{i}\right)=r_{i}+R_{i-1}=l$ hence

$$
\theta_{i} \geq \frac{e^{(m-1) b_{0}}\left(1+\frac{R_{i-1}}{r_{i}}\right)^{m}}{e^{(m-1) b_{0}}\left(1+\frac{R_{i-1}}{r_{i}}\right)^{m}-1} \theta_{i-1}
$$

$R_{i-1}=\sum_{j=0}^{i-1} 2.2^{b^{j}} \leq 2 \sum j=0^{i-1} 2^{\left[b^{j}\right]+1} \leq 2.2^{\left[b^{i-1}\right]+2}$ where [ ] denotes the integer party; this implies that $\frac{R_{i-1}}{r_{i}} \leq 1$ and goes to zero at infinity.

Since the function

$$
f(x)=\frac{e^{(m-1) b_{0}}(1+x)^{m}}{e^{(m-1) b_{0}}(1+x)^{m}-1}
$$

is nonincreasing on $[0,1]$ we have:

$$
\frac{2^{m} e^{(m-1) b_{0}}}{2^{m} e^{(m-1) b_{0}}-1} \leq f(x) \leq \frac{e^{(m-1) b_{0}}}{e^{(m-1) b_{0}}-1}
$$

hence

$$
\theta_{i} \geq \frac{2^{m} e^{(m-1) b_{0}}}{2^{m} e^{(m-1) b_{0}}-1} \theta_{i-1}
$$


which means :

$$
\theta_{i} \geq a^{i} \theta_{0}
$$

where

$$
a=\frac{2^{m} e^{(m-1) b_{0}}}{2^{m} e^{(m-1) b_{0}}-1}
$$

and $\theta_{0}=\operatorname{vol} B(p, 2)$

Let $R$ be a sufficient large positive number; there exists $i$ such that $r_{i-1} \leq R \leq r_{i}$, otherwise

$$
\begin{gathered}
r_{i}=2^{b^{i}} \Rightarrow i=\frac{\ln \left(\frac{\ln r_{i}}{\ln 2}\right)}{\ln b} \geq \frac{\ln (\ln R)}{\ln b} \\
\theta_{i} \geq C . a^{\frac{\ln \left(\frac{\ln r_{i}}{\ln 2}\right)}{\ln b}} \geq C \cdot a^{\frac{\ln (\ln R)}{\ln b}}=C(\ln (R))^{\rho}
\end{gathered}
$$

Where $C, C_{1}$ are positive constants and $\rho=\frac{\ln a}{\ln b}>0$

Those results give a hope for showing the following conjecture due to S.H.Zhu and which is a version of Grove and Peterson conjecture's:

Conjecture: Given $c>0$, do there exist constants $\epsilon(m, c)$ and $R(m, c)$ such that if

$$
K(x) \geq-\lambda(d(p, x)), \operatorname{Vol}(B(p, r)) \geq c r^{m},
$$

and

$$
\int_{0}^{\infty} t \lambda(t) d t \leq \epsilon
$$

then any metric ball of radius $\mathrm{r}$ is contractible in the cocentric ball of radius $R . r$ ? Is $M$ diffeomorphic to $\mathbf{R}^{m}$ ?

\section{Bibliography}

[Ab1] U.Abresch, Lower curvature bounds, Toponogov's theorem and bounded topologyI, Ann. Sci.Ecole Norm. Sup. 18, (1985) 651-670.

[Ab2] U.Abresch, L ower curvature bounds, Toponogov's theorem and bounded topologyII, Ann. Sci. Ecole Norm. Sup.20(1987)475-502.

[AG] U.Abresch and D.Gromoll, On complete manifolds with nonnegative Ricci curvature, J.Amer. Math. Soc. 3 (1990) 355-374. 
[Ca] M. I. Cai, Ends of Riemannian manifolds with nonnegative Ricci curvature outside of compact set, Bull. Amer.Math. Soc. 24 (1991), 371-377.

[CG1] J. Cheeger and D. Gromoll, The splitting theorem for manifolds of nonegative Ricci curvature, J. Diff. Geometry 6 (1971),119-128.

[CG2] J. Cheeger and D. Gromoll, On the structure of complete manifolds of nonnegative curvature, Ann. Math. (2) 96 (1972) 413-443.

[Ch] J. Cheeger, Critical points of distance functions and applications to geometry, Lectures notes 1504 (1991) 1-38.

[Ka] A. Kasue, Harmonic functions with growth conditions on a manifold of asymptotically nonnegative curvature Adv.Stud.Math.18-I (1990) 283301.

[Zh] S. H. Zhu, A volume comparison theorem for manifolds with asymptotically nonnegative curvature and its applications, Amer. J. Math. 116 (1994), 669-682.

Faculté des sciences

Niamey

Niger

E-mail: bmahaman@yahoo.fr

Recibido: 2 de Noviembre de 1998

Revisado: 10 de Mayo de 1999 